\title{
Advanced Technologies in Wireless Internet and Communications Networks (Editorial for WICON 2011 Special Issue)
}

\author{
Pinyi Ren • Jun Cai $\cdot$ Zhou Su
}

Published online: 25 October 2012

(C) Springer Science+Business Media New York 2012

Over the last two decades, wireless internet and communications networks have experienced an explosive growth in both the diversity of the techniques and its range of applications. Besides the traditional mobile communications services, current mobile communications networks can also provide internet access services. In order to provide higher data transmission rate, the 4th generation mobile communications systems, i.e., IMT-Advanced, have been developed and many advanced technologies, such as MIMO and OFDM, have been applied. The constantly developing wireless communications technologies also have enabled the extensive deployment of distributed wireless networks, such as the Ad Hoc networks. This kind of networks has applications in many important areas, such as military, healthcare, environment monitoring, and manufacturing. Due to the absence of central controllers, the distributed networks face many challenges. In recently years, radio spectrum has becoming exhausted because of the growing demands for wireless broadband communications. However, the traditional static spectrum allocation policy has prevented the spectrum utilization efficiency from further increasing. In order to overcome the spectrum shortage problem, cognitive wireless network, in which cognitive radio technique is applied, have drawn a great of research attentions. In the future wireless internet and communications networks,

P. Ren $(\bowtie)$

Xi'an Jiaotong University,

Xi'an, China

e-mail: pyren@mail.xjtu.edu.cn

J. Cai

University of Manitoba,

Manitoba, Canada

Z. Su

Waseda University,

Tokyo, Japan many advanced technologies, such as MIMO, OFDM, cognitive radio, distributed resource allocation/management, distributed admission control, etc., will be applied to satisfy the increasing demands for broadband wireless services.

Our objectives for this special issue of ACM/Springer Mobile Networks \& Applications are to collate and disseminate the most relevant achievements of research work on wireless network architectures, wireless access technologies, distributed admission control, advanced resource allocation/management, QoS guarantees, etc. In response to this call for papers, we have received a total of xx high-quality submissions, and 6 papers have been selected for publication after a rigorous review process.

The first paper "Optimal Resource Allocation for Spectrum Sensing Based Cognitive Radio Networks with Statistical QoS Guarantees" by Y. Wang, P, Ren, Q. Du, and C. Zhang proposes an optimal resource allocation scheme for statistical QoS provisioning in spectrum sensing based cognitive radio networks subject to the average transmit and interference power constraints. The second paper "The Master-slave Stochastic Knapsack Modeling for Fully Dynamic Allocation" by S. Zhang, F. Yang, M. Zhao, and W. Zhou models the fully dynamic spectrum allocation scheme as a master-slave stochastic knapsack process, analyzes the equilibrium state transition, and derives the probability of blocking and forced termination for the secondary user. The third paper "A Method for Classifying Packets into Networks Flows Based on GHSOM" by H. Shi, T. Hamagami, H. Xu, P. Yu, and Y. Wu develops an intelligent network flow identifying method based on the neural network algorithm and suggests a new normalization for changing the raw network traffic to learn input vectors. The forth paper "Performance Improvement of the Precise Paging in CoLAC for $2 \mathrm{G} / 3 \mathrm{G}$ Convergence Core Networks with Path Diversity" by Y. Su and X. Feng evaluates the system performance metrics in terms of handover success rate and 
network completion rates through the implementation over the realistic $2 \mathrm{G}$ and $3 \mathrm{G}$ systems. The fifth paper "A new Cooperative Spectrum Sensing Scheme for Cognitive Ad Hoc Networks" by Y. Du, H. Li, W. Lin, L. Liu, X. Wang, S. U. Khan, and S. Wu introduces a new guard-resident cooperative spectrum sensing scheme for a cognitive ad-hoc network, in which only part of the cognitive nodes named guard nodes perform the spectrum sensing. The sixth paper "FAST: Fuzzy Decision-Based Resource Admission Control Mechanism for MANETs" by Y. Bai, X. Zhu, X. Shao, and W. Yang combines resource admission control technologies and intelligent methods and proposes a fuzzy decision-based resource admission control mechanism for MANETs (FAST), which can fast respond to dynamic topology changes and unstable link status.

The Guest Editor would like to thank all the authors who have contributed their time and effort submitting their excellent works in response to the call for papers, regardless of whether their works have been accepted in this Special Issue or not due to space limitations. The Guest Editors would also like to thank all the reviewers for their valuable suggestions to the authors on improving the content and presentation of their papers.

Dr. Pinyi Ren received the Ph.D. degree in Electronic and Communications System, the M.S. degree in Information and Communications Engineering, the B.S. degree in Information and Control Engineering, in 2001, 1997, and 1994, respectively, all from Xi' an Jiaotong University, China. He is currently a Professor and the Department Head of Information and Communications Engineering Department, Xi'an Jiaotong University, China. He was appointed as the Vice Program Director for the Key Project "Spectrum Resource Sharing Based Wireless Communications Systems" of the National Hi-Tech Research and
Development (863) Program of China during the "Eleventh Five-Year Plan" (2006-2010). He has been the PIs and/or Co-PIs for over ten national projects sponsored by the Chinese Government; he has been the PIs and/or PIs for more than ten projects sponsored by Chinese Engineering Academy, by the Shaanxi Province, by the City of Xi'an, by Xi'an Jiaotong University, and by several companies. He has published over 60 technical papers on international Journals and conferences. He received the Best Letter Award of IEICE Communications Society 2010.

Dr. Jun Cai received the B.Sc. (1996) and the M.Sc. (1999) degrees from Xi'an Jiaotong University (China) and Ph.D. degree (2004) from University of Waterloo, Ontario (Canada), all in electrical engineering. From June 2004 to April 2006, he was with McMaster University as NSERC Postdoctoral Fellow. Since July 2006, he has been with the Department of Electrical and Computer Engineering, University of Manitoba, Canada, where he is an Associate Professor. His current research interests include multimedia communication systems, mobility and resource management in $3 \mathrm{G}$ beyond wireless communication networks, cognitive radios, and ad hoc and mesh networks. Dr. Cai serves as the Technical Program Committee Co-Chair for IEEE VTC 2012-Fall Wireless Applications and Services Track, IEEE Globecom 2010 Wireless Communications Symposium, and IWCMC 2008 General Symposium, the Publicity Co-Chair for IWCMC 2010, 2011 and 2013, and the Registration Chair for the First International Conference on Heterogeneous Networking for Quality, Reliability, Security and Robustness (QShine) 2005. He also served the editorial board of Journal of Computer Systems, Networks, and Communications. Dr. Cai received the Rh Award for outstanding contributions to research in applied sciences in 2012 from University of Manitoba, and Outstanding Service Award from IEEE Globecom 2010.

Dr. Zhou Su is an associate professor of Waseda University. Japan. His research interests include on Network Traffic, Internet Architecture. Contents Delivery, Mobile Multimedia, WWW, P2P, Overlay Network. He has published more than 50 journal/conference articles and book chapters. He has served TPC members of numerous IEEE conferences including INFOCOM, Globecom, ICC, VTC and WCNC. He is a member of IEICE and IEICE. 